\title{
Quantitative NMR Approach to Optimize the Formation of Chemical Building Blocks from Abundant Carbohydrates
}

\author{
Elliot, Samuel Gilbert; Tolborg, Søren; Sádaba, Irantzu; Taarning, Esben; Meier, Sebastian
}

Published in:

ChemSusChem (Print)

Link to article, DOI:

$10.1002 /$ cssc. 201700587

Publication date:

2017

Document Version

Peer reviewed version

Link back to DTU Orbit

Citation (APA):

Elliot, S. G., Tolborg, S., Sádaba, I., Taarning, E., \& Meier, S. (2017). Quantitative NMR Approach to Optimize the Formation of Chemical Building Blocks from Abundant Carbohydrates. ChemSusChem (Print), 10(14), 29902996. https://doi.org/10.1002/cssc.201700587

\section{General rights}

Copyright and moral rights for the publications made accessible in the public portal are retained by the authors and/or other copyright owners and it is a condition of accessing publications that users recognise and abide by the legal requirements associated with these rights.

- Users may download and print one copy of any publication from the public portal for the purpose of private study or research.

- You may not further distribute the material or use it for any profit-making activity or commercial gain

- You may freely distribute the URL identifying the publication in the public portal 


\section{Quantitative NMR Approach to Optimize the Formation of Chemical Building Blocks from Abundant Carbohydrates}

\begin{abstract}
Samuel G. Elliot, ${ }^{[\mathrm{a}]}$ Søren Tolborg, ${ }^{[\mathrm{b}]}$ Irantzu Sádaba
The future role of biomass-derived chemicals relies on the formation of diverse functional monomers in high yields from carbohydrates. Recently, it has become clear that a series of a-hydroxy acids, esters and lactones can be formed from carbohydrates in alcohols and water using tin-containing catalysts such as Sn-Beta. These compounds are potential building blocks for polyesters with additional olefin and alcohol functionalities. We employ an NMR approach to identify, quantify and optimize the formation these building blocks in the chemocatalytic transformation of abundant carbohydrates by Sn-Beta. Record yields of the target molecules can be achieved by obstructing competing reactions through solvent choice.
\end{abstract}

The need to establish more sustainable ways to obtain the chemicals needed by society for the production of food, materials, fuels and energy is widely recognized. The current chemical industry is based on the availability of a small number of petroleum-derived building blocks. Biomass can be utilized to provide access to both existing and new types of building blocks and research into this area has steeply increased during the last decade. It is important for these chemicals to be accessible at low cost and at the same time to have useful properties, in order to be utilized commercially. Therefore, direct conversion of sugars to a target chemical by heterogeneous catalysis, offers the best chance of lowering the process costs. ${ }^{[1-4]}$ A plethora of reaction products have been identified in the acid-catalyzed conversion of C5 and C6 carbohydrates. ${ }^{[5]}$ Amidst them acyclic a-hydroxy esters and acids have recently emerged as an attractive group of bio-monomers (Scheme 1).

For the use in biopolymer applications, the production, polymerization and upgrading of the $\mathrm{C} 3$ building block lactate (7) and the C4 building block vinyl glycolate (8) have previously been demonstrated. ${ }^{[3,6-11]}$ Recently, the production and polymerization of the C5 building block 2,5-dihydroxy-3pentenoic acid methyl ester (Me-1) as well as the formation of the C6 building block trans-2,5,6-trihydroxy-3-hexenoic acid methyl ester (Me-12) have been described (Table 1). ${ }^{[12-14]}$

[a] S. G. Elliot, Dr. S. Meier

Department of Chemistry

Technical University of Denmark

Kemitorvet, Bygning 207, 2800 Kgs. Lyngby, Denmark

E-mail: semei@kemi.dtu.dk

[b] Dr. S. Tolborg, Dr. I.Sádaba, Dr. E. Taarning,

Haldor Topsøe A/S, Haldor Topsøes Alle 1, 2800 Kgs. Lyngby, Denmark.

Supporting information for this article is given via a link at the end of the document.

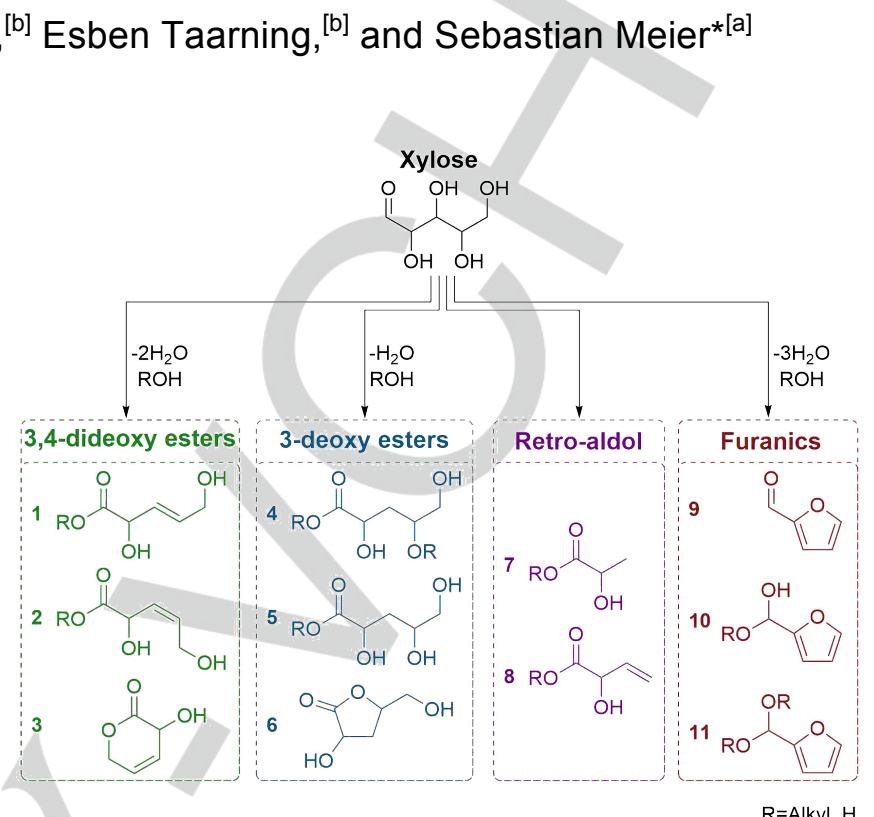

Scheme 1. Overview of major pathways in the catalytic conversion of xylose by $\mathrm{Sn}$-Beta and the major products identified in the reaction mixtures at high temperature $\left(>100^{\circ} \mathrm{C}\right)$. The products are shown for reactions in alcohol $(R=$ alkyl) or water $(R=H)$ and are grouped (from left to right) as 3,4-dideoxy esters, 3-deoxy esters, retro-aldol products and furanics.

Table 1. Conversion of $\mathrm{C} 4, \mathrm{C} 5$ and $\mathrm{C} 6$ carbohydrates by Sn-Beta to homologous 3,4-dideoxy esters with declining yield.

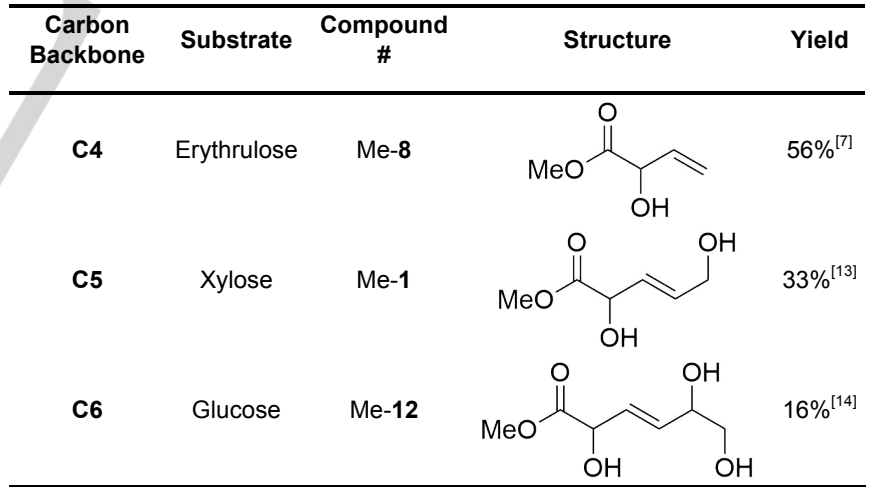

The conversion of C5 and C6 carbohydrates to the acyclic unsaturated $\alpha$-hydroxy compounds 1 and 12 has been achieved using Sn-Beta, in methanol ${ }^{[13-14]}$ or water ${ }^{[12]}$ at temperatures above $100{ }^{\circ} \mathrm{C}$ in the absence of further additives. A Sn-Beta zeolite synthesized under hydrothermal conditions with hydrofluoric acid as the mineralizing agent has so far proven most efficient for this reaction. ${ }^{[12-14]} \mathrm{C} 5$ and C6 carbohydrates yield around $30 \%$ for C5 carbohydrate substrate (Me-1) and around $15 \%$ for $\mathrm{C} 6$ carbohydrate substrate (Me-12), respectively, while the yields for the formation of the $\mathrm{C} 4$ analogue vinyl glycolate methyl ester (Me-8) can reach above 50\% (Table 1). ${ }^{[7,13-14]}$ Addition of alkali salts has been shown to reduce those yields and to strongly enhance the formation of smaller compounds such as methyl lactate, instead. ${ }^{[8]}$ 
The detection and optimization of carbohydrate conversion to new chemicals requires consideration of the optimum analytical approaches employed. Thus, methods that rely on the use of reference standards for identification and quantification, including liquid and gas chromatography, may be challenging to apply in work streams towards new chemicals. In cases where commercial standards are unavailable, either production of standards or calculations to estimate response factors are necessary. ${ }^{[10]}$ In contrast, methodologies that combine detailed structural information and the possibility of obtaining an accurate quantitative signal may be more valuable in the push towards bio-based economies.

In the current study, we combine in-situ NMR spectroscopy for the identification of reaction products and an accurate quantitative NMR (qNMR) methodology to assess solvent effects in the Sn-Beta catalyzed conversion of abundant carbohydrates. The methodology operates on a timescale comparable to commonly used chromatography methods but avoids instrumental response factors altogether. Identification of new chemicals in situ makes their purification and characterization obsolete. Water and a series of short-chain alcohols are used as solvents that provide sufficient substrate solubility for the carbohydrates. The change of solvents from methanol and water to longer-chain alcohols is motivated by the varying physicochemical solvent properties and by the stoichiometric participation of the solvent as a nucleophile in the reaction. Thus, the choice of solvent will affect the formation of alkyl-glycoside and acetal type intermediates during the reaction, modulate the microenvironment and Lewis acid properties of tin active sites in the stannosilicates and alter molecular dynamics and energetics in the reaction path. ${ }^{[9-10]}$ Hence, solvent variation is a means of optimizing operation conditions towards increased profitability of bioprocesses.

For the analysis of solvent effects, we use optimized spectra on complex reaction mixtures without prior purification or sample pre-treatment in protonated organic solvents. The approach does not rely on the availability of purified reference compounds. Changes in product structure due to reaction with the solvent do not critically complicate product identification and quantification for the 11 main reaction products.

\section{Results and Discussion}

Detection of products in reaction mixtures without relying on reference compounds

NMR spectroscopy is widely used for chemical structure elucidation and mixture analysis. The use of NMR spectroscopy for component identification and quantification is particularly well developed for biological samples, mostly biofluids, extracts and foods, but has also gained popularity within biomass conversion, for instance in the study of carbohydrate isomerization reactions $^{[15-17]}$ or of lignin structure and depolymerization reactions ${ }^{[18]}$ Here, we use a suite of homo- and heteronuclear assignment spectra for the detection and identification of carbohydrate degradation products in situ. Specifically, DQFCOSY, TOCSY, ${ }^{1} \mathrm{H}^{13} \mathrm{C}$ HMBC, conventional and edited ${ }^{1} \mathrm{H}^{13} \mathrm{C}$ $\mathrm{HSQC}$ as well as ${ }^{1} \mathrm{H}-{ }^{13} \mathrm{C}$ HSQC-TOCSY spectra were employed for compound identification. Band-selective ${ }^{13} \mathrm{C}$ excitation ${ }^{[19]}$ and optimized decoupling sequences ${ }^{[20-21]}$ were used to suppress non-informative signals and artefacts and to obtain higher quality ${ }^{1} \mathrm{H}-{ }^{13} \mathrm{C}$ spectra. NMR spectroscopy has notorious shortcomings in the detection of heteroatoms beyond protons and carbons, but this problem was negligible for reactions involving carbohydrate fragmentation or dehydration, as oxygen positions could be inferred from ${ }^{13} \mathrm{C}$ chemical shifts. At the same time, the detection of discrete signals for individual atomic positions by NMR spectroscopy allows the distinction of isomers. Such distinction of isomers is crucial, as several potential products in carbohydrate dehydration cannot be distinguished based on their mass alone.

Major products that were identified in reaction mixtures produced from xylose at $160^{\circ} \mathrm{C}$ using Sn-beta zeolite are shown in Scheme 1. These compounds were identified through de novo structure determination and ${ }^{1} \mathrm{H} /{ }^{13} \mathrm{C}$ chemical shift assignments in unpurified reaction mixtures in six different protic solvents (water, methanol, ethanol, $n$-propanol, iso-propanol and $n$-butanol). This approach was aided by the use of high-field NMR instrumentation (18.7 Tesla magnets) equipped with cryogenically cooled detection electronics to reduce electronic noise approximately 3 -fold. The identified reaction products derive from pathways including $\mathrm{C}-\mathrm{C}$ bond breakage in retro-aldol reactions to yield $\mathrm{C} 2, \mathrm{C} 3$ and $\mathrm{C} 4$ fragments, which may subsequently undergo dehydration to various $\alpha$-hydroxy esters (7-8). Alternatively, direct dehydration of the C5 compound to $\alpha$ hydroxy, 3-deoxy or 3,4-dideoxy esters (1-6) or triple dehydration to furanics (9-11) can occur. The most interesting of these products may be the trans-2,5-dihydroxy-3-pentenoic acid alkyl ester (1). We recently showed that this prospective chemical building block could be co-polymerized enzymatically in a selective 1,5 polymerization reaction with ethyl 6-hydroxyhexanoate, yielding polymers that could be specifically functionalized at the secondary alcohol group or at the olefinic bond of the monomer. ${ }^{[13]}$ Thus, 2,5-dihydroxy-3-pentenoic acid alkyl esters could form the platform for a vast variety of functional materials derived from C5 carbohydrates.

Standard reaction conditions were defined based on optimizations of trans-2,5-dihydroxy-3-pentenoic acid methyl ester (Me-1) formation in methanol. ${ }^{[13]}$ In these studies, a SnBeta catalyst was found to be most effective for the formation of $\mathrm{Me}-1$, and therefore such a catalyst was also employed in this study of solvent effect.

One-dimensional ${ }^{13} \mathrm{C}$ NMR spectra of reaction mixtures carbonyl regions obtained in various solvents are shown in Figure 1, assigned compounds corresponding to the spectral signals are shown in Scheme 1. In these reaction mixtures, careful inspection allowed the identification of the minor cis-2,5dihydroxy-3-pentenoic acid alkyl ester (2) in addition to the dominant trans-form (1). The ${ }^{1} \mathrm{H}-{ }^{13} \mathrm{C}$ spectral region showing both molecules and their chemical shift assignments in methanol is displayed in Figure 2. Long-range correlations through ${ }^{2} J_{\mathrm{HC}}$ couplings across the double bond are indicated by white lines and serve to identify the connected signals as parts of the same NMR spin system. The cis- and trans-configurations were identified by their characteristic ${ }^{3} \mathrm{~J}_{\mathrm{HH}}$ scalar couplings across the olefinic bond and by the characteristic signal shifts to lower frequencies for carbons adjacent to the cis-double bond. This 
cis-isomer was identified as the minor isomer in all solvents. Together with these products, 6 additional 3-deoxy ester/acid compounds were identified in the carbonyl region shown in Figure 1. These signals cluster within different spectra regions in accordance with their functionality: olefinic esters $(1-3,8)$ at 172

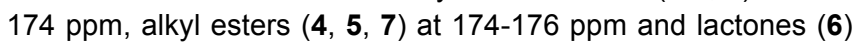
above $177 \mathrm{ppm}$. Further inspection of the ${ }^{13} \mathrm{C}$ NMR spectra of

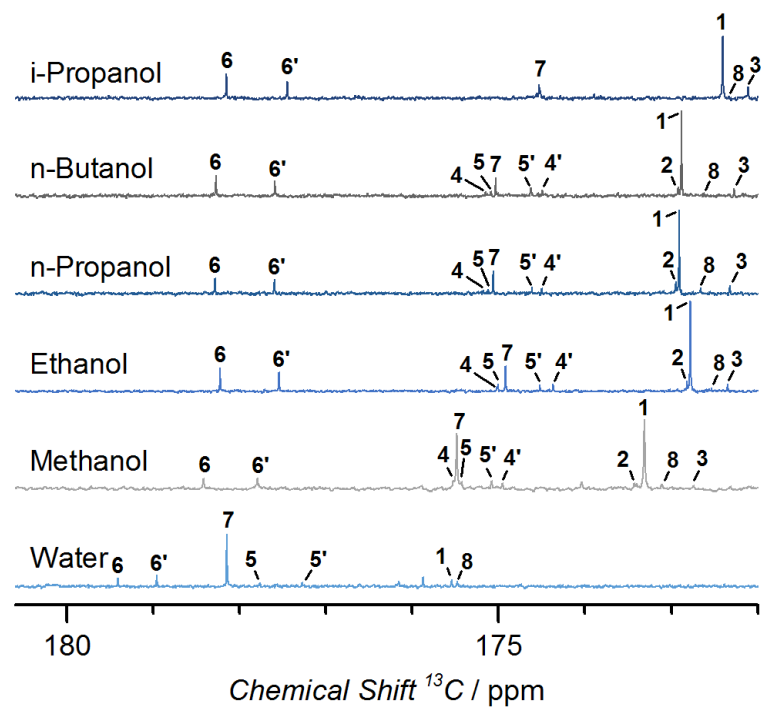

Figure $1 .{ }^{13} \mathrm{C}$ NMR spectra after converting $360 \mathrm{mg} \mathrm{D}$-xylose with $180 \mathrm{mg} \mathrm{Sn-}$ Beta in $5 \mathrm{~mL}$ solvent. The carboxyl spectral region is shown and signals are assigned to the molecules shown in Scheme 1; an apostrophe denotes the diastereomer of a compound. Chemical shifts are relative to deuterated methanol (added to $10 \% \mathrm{v} / \mathrm{v}$ ) set to $47.85 \mathrm{ppm}$ for all solvents.

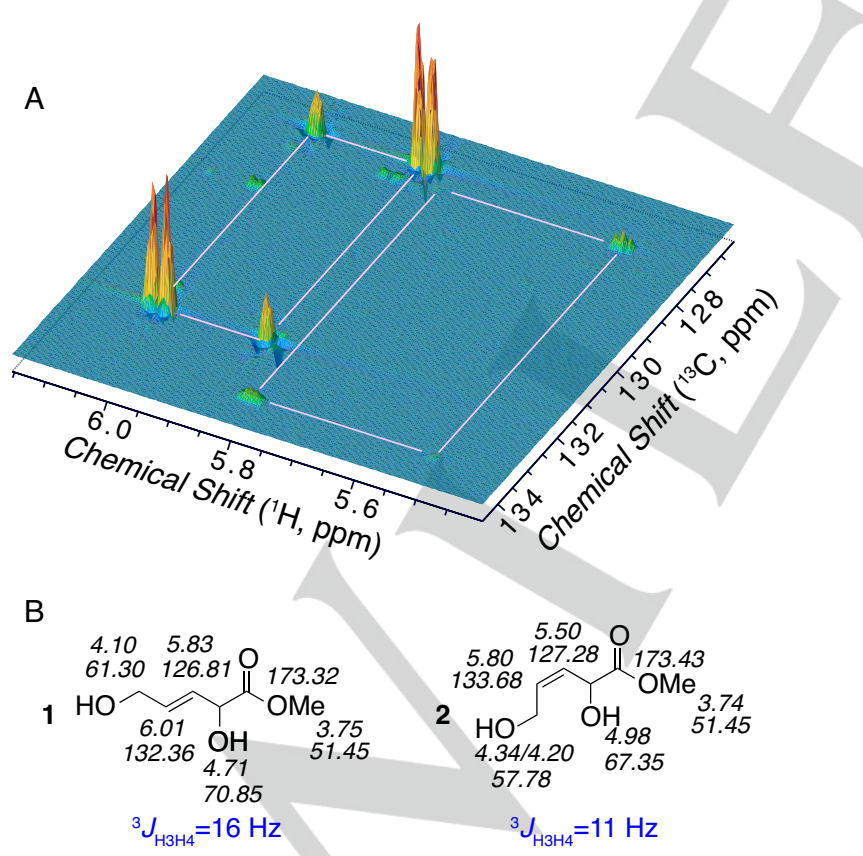

Figure 2. (A) ${ }^{1} \mathrm{H}_{-}{ }^{13} \mathrm{C}$ HSQC spectral region displaying the olefinic signals of compound 1 (trans-isomer) and 2 (cis-isomer) in a reaction mixture. ${ }^{2} \mathrm{~J}_{\mathrm{CH}}$ correlations across the double bond are indicated by thin white lines. (B) Chemical shift assignments of compound 1 (trans-isomer) and compound 2 (cis-isomer).
Figure 1 indicates that rather dramatic compositional changes result from changing the solvent from water to alcohol. Furthermore, it was possible to distinguish the diastereomers of compounds 4-6 signified as by an apostrophe.

Quantification of bio-based chemicals in reaction mixtures Reliable quantifications of the compound series shown in Scheme 1 were subsequently pursued by quantitative NMR (qNMR) spectroscopy. As qNMR can operate at the highest level of quantitative measurement ${ }^{[22-23]}$ the method is free of empirical factors in the uncertainty analysis of the experiment and signal areas are directly proportional to the concentration of atoms contributing to the signal.

Cryogenically cooled detection electronics and high-field instrumentation enabled absolute quantifications of reaction mixtures by quantitative $1 \mathrm{D}^{13} \mathrm{C}$ NMR spectroscopy at natural ${ }^{13} \mathrm{C}$ isotope abundance $(1.11 \%)$. Experiments that only use inverse gated decoupling were used to avoid enhancement of the ${ }^{13} \mathrm{C}$ signals by the nuclear Overhauser effect. ${ }^{[24]}$ Crude reaction mixtures, subjected to quantitative ${ }^{13} \mathrm{C}$ NMR spectroscopy, contained dimethyl sulfoxide as an internal standard for absolute quantification. Dilute dimethyl sulfoxide showed good recovery after reactions without detectable degradation, while being miscible with the solvents tested in this study and did not result in ${ }^{1} \mathrm{H}$ or ${ }^{13} \mathrm{C}$ NMR signal overlap with analyte signals. Therefore, dimethyl sulfoxide was chosen and deemed preferable to compounds like mesitylene, dioxane, glycerol or other alditols tested for the spectroscopic characterization of reaction mixtures formed in catalytic carbohydrate conversion.

Quantitative 1D ${ }^{13} \mathrm{C}$ NMR rather than the more commonly used ${ }^{1} \mathrm{H}$ NMR spectroscopy was employed for quantification, as it provides excellent signal resolution and sharp non-split signals. Protonated carbon positions were generally used for quantification due to their shorter $\mathrm{T}_{1}$-NMR relaxation times, yielding quantitative signal for inter-scan relaxation delays on the sub-minute timescale. As an alternative, improved ${ }^{13} \mathrm{C}$ NMR spectra could be obtained upon addition of $1 \mathrm{mM} \mathrm{GdCl}_{3}$ as a relaxation agent to the NMR sample, shortening the carbonyl ${ }^{13} \mathrm{C}$ $\mathrm{T}_{1}$ relaxation time at room temperature and $18.7 \mathrm{~T}$ magnetic field to the $\sim 1$ second time scale. The reduced ${ }^{13} \mathrm{C} \mathrm{T}_{1}$ relaxation time permits the accurate measurement of ${ }^{13} \mathrm{C}$ NMR signal areas also for quaternary carbons without the need for long inter-scan recycle delays. The signal-to-noise ratio obtained within one hour of experiment time per sample translated to an estimated error of determination of $0.2-1.0 \%$ for product yields. Such small experimental uncertainty was validated by performing the analyses for reaction mixtures in all six different solvents in replicate, yielding near-identical ${ }^{13} \mathrm{C}$ NMR spectra for repetitions in each solvent (Figure S1).

\section{Solvent effect on Sn-Beta catalyzed reaction}

Quantifications of the eight major compounds in non-purified reaction mixtures in different solvents are displayed in Figure 3. Various trends become evident upon variation of the solvent. Upon increasing the alkyl chain of the alcohol solvent, the amount of retro-aldol products (7-8) decreases, consistent with previously reported observations. ${ }^{[7]}$ Simultaneously, an increased yield of 3,4-dideoxy esters (1-3) and related 3-deoxy esters (4-6) is observed. Notably, all longer chain alcohols 
tested gave higher yields of 3,4-dideoxy esters than methanol did, even at conditions that were specifically optimized for reactions in methanol. The formation of 3,4-dideoxy esters was highest in ethanol with yields at around $42 \%$ (38\% trans (1) and $4 \%$ cis (2-3) form) and thus 1.2-fold higher than in methanol. Consistently increased yields were also found for the lactone (6), yielding $15 \%$ in ethanol as compared to $8 \%$ in methanol. Overall, the 3-deoxy and 3,4-dideoxy esters (1-6) account for $66 \%$ of the carbon balance in ethanol and in iso-propanol. Quantifications of these related compounds are summarized in Table 2. Furfural derivatization to alkyl acetals predominates for methanol and is less pronounced in larger alcohols and especially in iso-propanol.

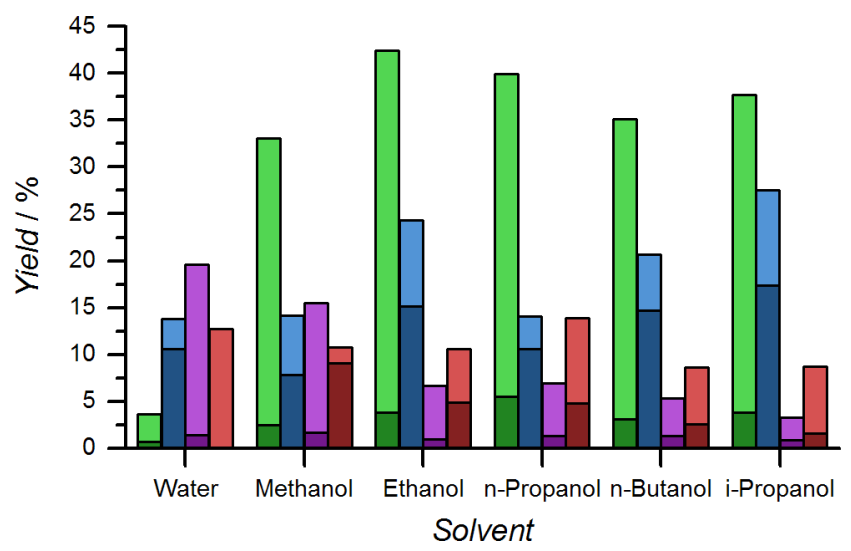

$$
\begin{aligned}
& \text { 3,4-dideoxy } 3 \text {-deoxy } \quad \text { Retro-aldol } \quad \text { Furanics } \\
& \square(1) \square(2-3) \square(4-5) \square(6) \square(7) \square(8) \square(9) \square(10-11)
\end{aligned}
$$

Figure 3. Product distribution of reactions in alcohols or water. The reactions were conducted with $180 \mathrm{mg}$ Sn-Beta, $360 \mathrm{mg} \mathrm{D-xylose,} 5 \mathrm{~mL}$ solvent and 50 mg dimethyl sulfoxide as internal standard, and run for 2 hours at $160{ }^{\circ} \mathrm{C}$ with $600 \mathrm{rpm}$ stirring. An experimental uncertainty within $\pm 1 \%$ was achieved in all cases. Legend numbers reference to the compounds in Scheme 1.

In all solvents, 3,4-dideoxy esters are primarily formed as the trans compound (1), with yields that are also higher than both the cis 3,4-dideoxy form (2-3) and the potentially related furfural forms (9-11) combined.

Table 2. Yield of 3,4-dideoxy compounds (1-3) and 3-deoxy compounds (4-6) from xylose in various solvents.

\begin{tabular}{ccccccc}
\hline Compound & Water & Methanol & Ethanol & $\begin{array}{c}\mathrm{n}- \\
\text { Propanol }\end{array}$ & $\begin{array}{c}\mathrm{n}- \\
\text { Butanol }\end{array}$ & $\begin{array}{c}\text { iso- } \\
\text { Propanol }\end{array}$ \\
\hline $\mathbf{1 - 3}$ & $4 \%$ & $33 \%$ & $42 \%$ & $40 \%$ & $35 \%$ & $38 \%$ \\
$\mathbf{4 - 6}$ & $14 \%$ & $14 \%$ & $24 \%$ & $14 \%$ & $21 \%$ & $28 \%$ \\
\hline \multirow{2}{*}{ Sum } & $17 \%$ & $47 \%$ & $66 \%$ & $54 \%$ & $56 \%$ & $66 \%$ \\
\hline
\end{tabular}

Reactions conducted in water exhibited a significantly altered composition than reactions conducted in alcohols, also exhibiting a greater tendency towards humin formation and catalyst discoloration. Thus, 3,4-dideoxy compounds are formed at lower levels than retro-aldol products, furanics and 3-deoxy compounds in water. A principal difference in the use of water and alcohol arises in the formation of free Brønsted acids as products in reactions conducted in water. Brønsted acids are known to catalyze a multitude of reactions including dehydration and acetalization. Due to this catalytic activity of Brønsted acids, their continuous formation will impact the catalytic system and alter the product distribution, thus rationalizing the principally different product composition in water and alcohols. Especially where the 3,4-dideoxy compounds are targeted, water is overall less useful as the solvent compared to the alcohols.

We finally note that reaction selectivity has been partially attributed to steric hindrance at the Sn active site in zeolite pores. ${ }^{[17]}$ In the current solvent study, the use of less nucleophilic and more bulky solvent increases the selectivity towards the formation of C5 lactones (compound 6). This change in selectivity is consistent with a favored intramolecular reaction rather than a reaction with the solvent for bulkier alcohols in Sn-Beta zeolite pores.

\section{Temperature effect in ethanol}

As improved yields of 3,4-dideoxy esters were achieved in other alcohols than in methanol, even under conditions that had been optimized for methanol, we evaluated the prospect for further improvements of the reaction using ethanol as the solvent. In the range of $140-160{ }^{\circ} \mathrm{C}$, the product selectivity was reasonably constant with similar yields of 3,4-dideoxy esters $(42 \%$, see Table S2). Overall, we find the formation of the 3-deoxy and 3,4dideoxy dehydration products (1-6) to be only weakly sensitive to temperature variation between 120 and $180^{\circ} \mathrm{C}$. Nevertheless, exploring solvent and temperature effects resulted in a combined yield above $40 \%$ for 3,4-dideoxy esters, an almost $10 \%$ increase relative to previously achieved yields in methanol (33\%, Table 1).

Temperature-dependent differences are detected in the formation of formation of the cyclic $\gamma$-lactone compound 6 relative to the open-chain 3-deoxy compounds 4 and $\mathbf{5}$. Lower temperatures favor the formation of compound $\mathbf{6}$ relative to the open-chain compounds $\mathbf{4}$ and $\mathbf{5}$. This observation is consistent with an increasing fraction of acyclic forms due to endothermic $\gamma$ lactone solvolysis to compound 5. ${ }^{[25]}$ Tabulated yields of the temperature study are collected in the SI (Table S2).

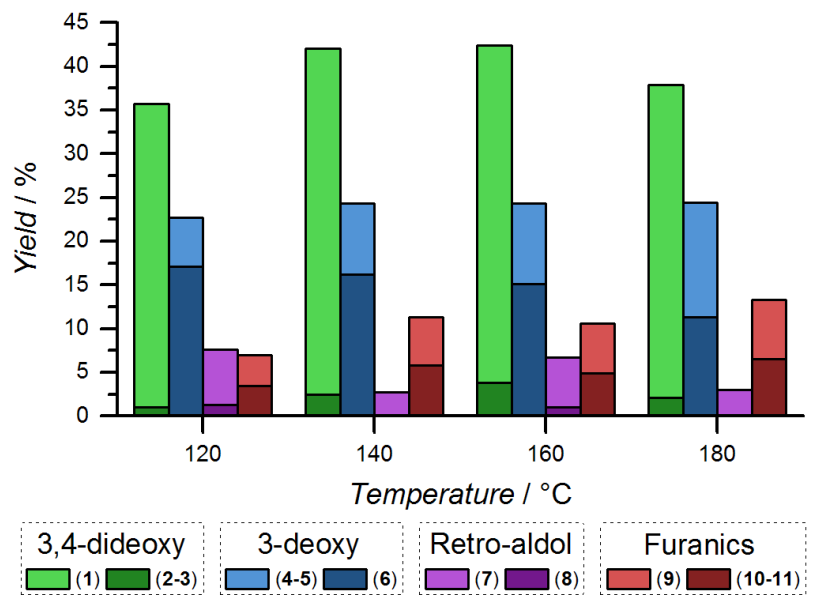

Figure 4. Product distribution of reactions between $120^{\circ} \mathrm{C}$ and $180{ }^{\circ} \mathrm{C}$ using ethanol as the solvent. The reactions were conducted using $180 \mathrm{mg} \mathrm{Sn-Beta,}$ $360 \mathrm{mg}$ D-xylose, $5 \mathrm{~mL}$ ethanol and $50 \mathrm{mg}$ dimethyl sulfoxide as internal standard, and run for 2 hours at the selected temperature. An uncertainty within $\pm 1 \%$ was achieved in all cases. Legend numbers reference to the compounds in Scheme 1. 


\section{Time-resolved reaction analysis}

Additional insight into solvent effects on the acyclic reaction of xylose to potential polyester building blocks was sought by tracking the reaction progress in water, methanol and ethanol over time. To this end, reactions were conducted under microwave heating for varying time and were subsequently analyzed by quantitative ${ }^{13} \mathrm{C}$ NMR spectroscopy (full data provided in Table S3). The experiments show continued formation of products even after full xylose conversion. This observation is attributed to the accumulation of glycoside intermediates ${ }^{[14]}$ some of which subsequently can be converted to the full range of products.

The formation of compound 1 , the main product, in methanol and ethanol, was tracked in an initial-rate experiment at $160{ }^{\circ} \mathrm{C}$ under quasi-steady state conditions as shown in Figure 5. Due to the initial surplus of substrate, pseudo-first order kinetics were obtained, resulting in a linear accumulation of product during the short initial time period shown in Figure 5. Although compound 1 formed with higher selectivity in ethanol $(39 \%)$ than in methanol $(31 \%)$ and water (approximately $5 \%$ ) in the kinetic experiments, the initial rate of formation is lower in ethanol than in methanol. This finding on the formation of compound 1 is paralleled by a higher rate of conversion of xylose in methanol than in ethanol and a lower rate of conversion in water (Table S3).

Kinetic profiles underline the complexity of the reaction pathway and hint at a combination of solvent effects. Solvent molecules compete with the substrate for binding to tin active sites. A weaker binding to these sites is expected for alcohols relative to water, leading to a better availability of active sites for the substrate in alcohols than in water. ${ }^{[26-27]}$ This rationalization may explain the lower rate of formation of compound 1 and concomitantly lower rate of xylose conversion observed in water. In the Sn-Beta catalyzed conversion of xylose in alcohol, the alcohol enters the catalytic cycle as a nucleophilic reactant. The lower rate of forming compound $\mathbf{1}$ in ethanol than in methanol is thus consistent with previous reports for C4 carbohydrate products suggesting that lower nucleophilicity and steric limitations may alter product selectivity towards the 3,4-dideoxy compound. ${ }^{[9]}$

In methanol and ethanol the formation of 1-6 is continuous during the reaction course (Figure S2). In contrast, the yield of compound 1 in water shows a maximum and subsequent decrease during the reaction, while maintaining a consistent collective increase of 3-deoxy and 3,4-dideoxy compounds (1-6). This finding indicates that some rehydration of compound 1 can occur in water, especially in the presence of accumulating Brønsted acidity. Formation of compound $\mathrm{H}-7$ occurs more rapidly in water than formation of compounds Me-7 and Et-7 in alcohols (Figure S3). Accumulation of compound 7 in water is followed by an increase in formation of compound 7 , indicative of an alteration of the mechanism due to an increase in Brønsted acidity. Final levels of furanics decrease slightly from water to methanol and ethanol (Figure 3). Lower carbon balance in water and the discoloring of the reaction mixture also indicate the formation of undetetcted polymeric species (humins). The distinct selectivity of Sn-Beta-catalyzed xylose conversion in water relative to alcohols can be ascribed to lower reactivity at the $\mathrm{Sn}$ active sites due to competing solvent absorption and to mechanistic effects of Brønsted acid formation. The formation of alkyl lactates is largely suppressed in longer alcohols, consistent with previous studies at conditions that were optimized for lactate formation. Here, maximum alkyl lactate yields had been reported to drop from $65 \%$ in methanol to approximately $35 \%$ in ethanol and $25 \%$ in isopropanol. ${ }^{[7]}$ Overall, the beneficial effect of ethanol relative to methanol in the formation 1-3 can be ascribed to the less nucleophilic and larger ethanol disfavoring side reactions.

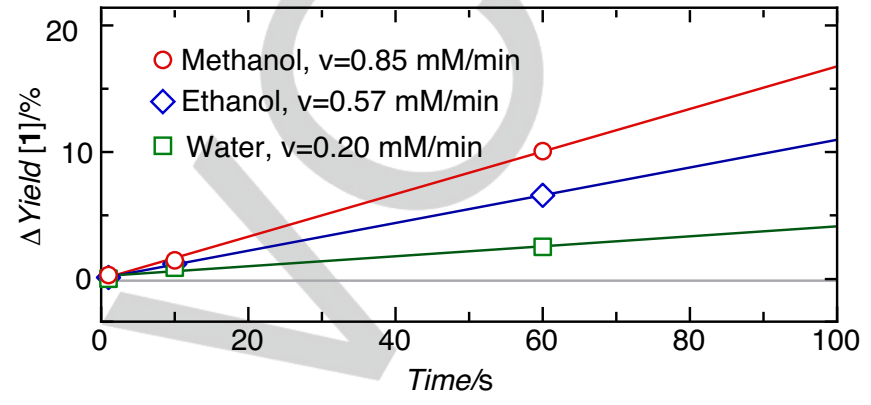

Figure 5. Initial rate experiments for the formation of product 1 from $360 \mathrm{mg}$ D-xylose $180 \mathrm{mg}$ by Sn-Beta at $160^{\circ} \mathrm{C}$ in $5 \mathrm{~mL}$ methanol, water and ethanol. The initial rate is lower in ethanol than in methanol, albeit a higher final concentration of $\mathbf{1}$ is reached in ethanol.

Improved yield of chemical building blocks from glucose

The abundance of glucose in biomass is even greater than that of xylose and glucose is the most abundant carbohydrate in nature. Glucose is available from starch and cellulose, which are both homopolymers composed of glucopyranose sub-units. Hexoses are able to form compound 12 (trans-2,5,6-trihydroxy3-hexenoic acid methyl ester, Figure $6 \mathrm{~A}$ ), the $\mathrm{C} 6$ analogue to compound 1, although in lower yields. In order to substantiate that the solvent effect discussed herein is applicable to the formation of chemical building blocks from C6 carbohydrates, as well, reactions using glucose as the substrate were performed.

The beneficial effect of ethanol relative to methanol in the formation of compound $\mathbf{1 2}$ is also valid for glucose, as can be seen from the NMR spectra of the olefinic region (Figure 6B). The solvent change from methanol to ethanol increases the formation of compound $\mathbf{1 2}$ approximately 1.2 -fold (from $13.8 \%$ to $16.0 \%$ under the standard reaction conditions used herein). The formation of the hexono-lactone equivalent of compound $\mathbf{6}$ also increases, from $16.6 \%$ to $19.4 \%$, while the formation of alkyl lactate 7 drops from $31.5 \%$ to $12.6 \%$. All of these effects closely follow trends observed for the conversion of xylose and are consistent with previous studies using homogeneous and heterogeneous tin catalysts on the less abundant acyclic C4 carbohydrate erythrulose. ${ }^{[9-10]}$ Thus, a shift towards improved selectivity for the formation of commercially interesting 3,4deoxy esters at the expense of retro-aldol cleavage can be achieved for a series of carbohydrates using Sn-Beta catalyst in longer alcohols than methanol. 
A

12<smiles>O=C(O)C(O)/C=C/C(O)CO</smiles>

B

Ethanol

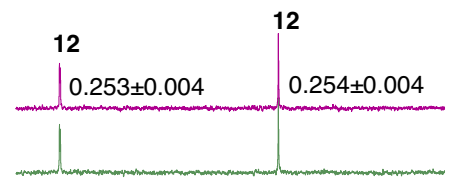

Methanol
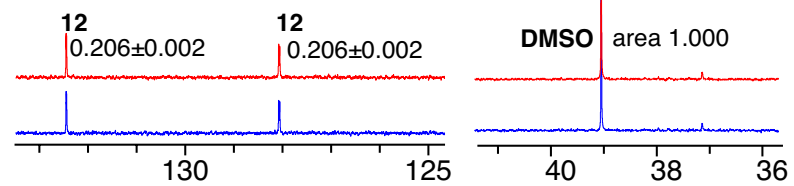

Chemical Shift $\left({ }^{13} \mathrm{C}, \mathrm{ppm}\right)$

Figure 6. (A) Structure of the $\mathrm{C} 6$ analogue of compound 1, trans-2,5,6trihydroxy-3-hexenoic acid methyl ester (12). (B) Spectral comparison of the areas of the olefinic carbon shifts of compound $\mathbf{1 2}$ in ethanol and methanol reaction solvents. Duplicate samples are shown, including errors of determination of signal areas relative to DMSO as an internal quantification standard.

\section{Conclusion}

In conclusion, we employ quantitative ${ }^{13} \mathrm{C}$ NMR for the detection and accurate quantification of previously unknown products in the conversion of abundant $\mathrm{C} 5$ and $\mathrm{C} 6$ carbohydrates by SnBeta, without using reference standard compounds. Identification and quantification are feasible in different solvents, thus permitting the elucidation of solvent effects on reaction selectivity. The exchanging of methanol for longer alcohols results in increased yields of C5 3,4-dideoxy esters, from 33\% in methanol to $42 \%$ in ethanol. This increase in yield can be ascribed to the less nucleophilic and larger ethanol disfavoring competing reactions. The improved yield of C5 3,4-dideoxy esters in ethanol relative to methanol and water correlates with a lower rate of formation, although the pathway towards 3,4dideoxy acids or esters is assumed to occur under kinetic control. $^{[9]}$ The formation especially of retro-aldol products is diminished in ethanol and longer alcohols. Nonetheless, the reaction pathways towards the formation of 3-deoxy and 3,4dideoxy compounds are broadly conserved in various protic solvents.

Simple alcohols have been considered as environmentally preferable solvents and ethanol has been described as preferable to methanol in terms of environmental, health and safety regulations. ${ }^{[28-29]}$ Hence, the formation of 2,5-dihydroxy-3pentenoic acid esters with $42 \%$ yield and of related 3-deoxyesters at combined yields near $66 \%$ from xylose in ethanol is encouraging for the development of environmentally benign processes in a future bio-based chemical industry.

\section{Experimental Section}

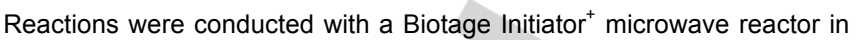
$5 \mathrm{~mL}$ glass reaction vials. Reactions were typically conducted with 180 $\mathrm{mg}$ Sn-Beta ( $\mathrm{Si} / \mathrm{Sn}=200$, hydrothermally synthesized), $360 \mathrm{mg}$ D-xylose, $5 \mathrm{~mL}$ solvent and $50 \mathrm{mg}$ dimethyl sulfoxide as internal standard.

Sn-Beta was produced according to the synthesis procedure described in the ESI, based on a modification of the procedure described by Valencia et $a l,{ }^{[30-31]}$ yielding the Sn-Beta catalyst with a Si/Sn ratio of 200. The product was confirmed by ICP $(\mathrm{Si} / \mathrm{Sn}=200)$, XRD ( ${ }^{*} \mathrm{BEA}$, Figure S4) and $\mathrm{N}_{2}$-adsorption (see ESI for details).

For discerning solvent effects on product composition and pathway usage, each sample was heated to $160{ }^{\circ} \mathrm{C}$ for 2 hours. $500 \mu \mathrm{L}$ aliquots were taken from each sample, $50 \mu \mathrm{L}$ of methanol- $\mathrm{d}_{4}$ (Sigma-Aldrich) was added and the mixture transferred to a $5 \mathrm{~mm}$ NMR tube for immediate analysis. In kinetic experiments, samples in water, methanol or ethanol were prepared in the composition detailed above and heated to $160^{\circ} \mathrm{C}$ for a variable duration ( $1 \mathrm{~s}, 10 \mathrm{~s}, 1 \mathrm{~min}, 10 \mathrm{~min}, 1 \mathrm{~h}, 2 \mathrm{~h}$ ). Samples were rapidly cooled with compressed air and $500 \mu \mathrm{L}$ aliquots were taken from each sample, mixed with $50 \mu \mathrm{L}$ of methanol- $\mathrm{d}_{4}$ and transferred to a $5 \mathrm{~mm}$ NMR tube.

All NMR spectra were acquired on an $800 \mathrm{MHz}$ Bruker Avance III NMR spectrometer equipped with a TCl CryoProbe and a SampleJet sample changer. $1 \mathrm{D}{ }^{13} \mathrm{C}$ NMR spectra were acquired by sampling $64 \mathrm{k}$ complex data points during and acquisition time of 1.36 seconds, and using an inter-scan recycle delay of 45 seconds. A pulse sequence with ${ }^{1} \mathrm{H}$ irradiation only during the signal acquisition was used to minimize distortions of signal integrals by the nuclear Overhauser effect. Protonated ${ }^{13} \mathrm{C}$ carbons were used for quantification and several carbon sites per molecule were used to improve the statistics of the signal area, using integrations in Bruker Topspin $3.5 \mathrm{pl} 5$ (see e.g. Figure 8). Standard DQF-COSY, TOCSY, ${ }^{1} \mathrm{H}-{ }^{13} \mathrm{C}$ HMBC, standard and edited ${ }^{1} \mathrm{H}-{ }^{13} \mathrm{C}$ HSQC as well as ${ }^{1} \mathrm{H}^{13} \mathrm{C}$ HSQC-TOCSY spectra were employed for compound identification in samples after evaporating the solvent overnight in a fume hood, prior to re-dissolution in deuterated solvents. Figure S6 demonstrates the accuracy of this method by comparison of a reaction sample with readily available standard compounds. Standard ${ }^{1} \mathrm{H}-{ }^{13} \mathrm{C}$ and edited HSQC as well as ${ }^{1} \mathrm{H}-{ }^{13} \mathrm{C}$ HSQC-TOCSY were used in protonated solvents to validate the assignments. All spectra were processed with ample zero filling in all spectral dimensions using Bruker Topspin 3.5 pl5.

\section{Acknowledgements}

This work was funded by the Innovation Fund Denmark (case number 5150-00023B) and by Grant 2013_01_0709 of the Carlsberg Foundation. $800 \mathrm{MHz}$ NMR spectra were recorded on the spectrometer of the DTU NMR center supported by the Villum foundation.

Keywords: carbohydrates $\cdot$ green solvents $•$ NMR $\cdot$ Sn-Beta • solvent effect

\section{References}

[1] Y. Román - Leshkov, J. N. Chheda, J. A. Dumesic, Science 2006, 312, 1933.

[2] J. B. Binder, R. T. Raines, J. Am. Chem. Soc. 2009, 131, 1979-1985 
[3] P. Gallezot, Chem. Soc. Rev. 2012, 41, 1538-1558.

[4] C. Li, Z. Zhang, Z. K. Zhao, Tetrahedron Lett. 2009, 50, 5403-5405.

[5] A. Corma, S. Iborra, A. Velty, Chem. Rev. 2007, 107, 2411-2502.

[6] E. Taarning, S. Saravanamurugan, M. S. Holm, J. Xiong, R. M. West, C. H. Christensen, ChemSusChem 2009, 2, 625-627.

[7] M. S. Holm, S. Saravanamurugan, E. Taarning, Science 2010, 328 602-605.

[8] S. Tolborg, I. Sádaba, C. M. Osmundsen, P. Fristrup, M. S. Holm, E. Taarning, ChemSusChem 2015, 8, 613-617.

[9] R. De Clercq, M. Dusselier, C. Christiaens, J. Dijkmans, R. I. lacobescu, Y. Pontikes, B. F. Sels, ACS Catal. 2015, 5, 5803-5811.

[10] M. Dusselier, P. Van Wouwe, F. de Clippel, J. Dijkmans, D. W. Gammon, B. F. Sels, ChemCatChem 2013, 5, 569-575.

[11] A. Sølvhøj, E. Taarning, R. Madsen, Green Chem. 2016, 18, 54485455.

[12] H. S. Chen, A. Wang, H. Sorek, J. D. Lewis, Y. Román - Leshkov, A. Bell, ChemistrySelect 2016, 1, 4167-4172.

[13] S. G. Elliot, C. Andersen, S. Tolborg, S. Meier, I. Sadaba, A. E. Daugaard, E. Taarning, RCS Adv. 2017, 7, 985-996.

[14] S. Tolborg, S. Meier, I. Sádaba, S. G. Elliot, S. K. Kristensen, S. Saravanamurugan, A. Riisager, P. Fristrup, T. Skrydstrup, E. Taarning, Green Chem. 2016, 18, 3360-3369.

[15] S. Saravanamurugan, A. Riisager, E. Taarning, S. Meier, Chem. Commun. 2016, 52, 12773-12776.

[16] S. Saravanamurugan, A. Riisager, E. Taarning, S. Meier, ChemCatChem 2016, 10.1002/cctc.201600783.

[17] S. Tolborg, S. Meier, S. Saravanamurugan, P. Fristrup, E. Taarning, I. Sádaba, ChemSusChem 2016, 10.1002/cssc.201600757.

[18] E. A. Capanema, M. Y. Balakshin, J. F. Kadla, J. Agric. Food Chem. 2004, 52, 1850-1860.
[19] C. Gaillet, C. Lequart, P. Debeire, J.-M. Nuzillard, J. Magn. Reson. 1999, 139, 454-459.

[20] E. Kupce, R. Freeman, G. Wider, K. Wüthrich, J. Magn. Reson., Ser. A 1996, 122, 81-84.

[21] Z. Zhou, R. Kümmerle, X. Qiu, D. Redwine, R. Cong, A. Taha, D. Baugh, B. Winniford, J. Magn. Reson. 2007, 187, 225-233.

[22] M. Weber, C. Hellriegel, A. Rueck, J. Wuethrich, P. Jenks, J. Pharm Biomed. Anal. 2014, 93, 102-110.

[23] H. Jancke, F. Malz, W. Haesselbarth, Accred. Qual. Assur. 2005, 10, 421-429.

[24] R. Freeman, H. D. W. Hill, R. Kaptein, J. Magn. Reson. 1972, 7, 327329.

[25] M. T. Pérez-Prior, J. A. Manso, M. del Pilar García-Santos, E. Calle, J. Casado, J. Org. Chem. 2005, 70, 420-426.

[26] W. N. P. van der Graaff, C. H. L. Tempelman, G. Li, B. Mezari, N. Kosinov, E. A. Pidko, E. J. M. Hensen, ChemSusChem 2016, 9, 31453149.

[27] J. R. Christianson, S. Caratzoulas, D. G. Vlachos, ACS Catal. 2015, 5, 5256-5263

[28] C. Capello, U. Fischer, K. Hungerbuhler, Green Chem. 2007, 9, 927934.

[29] M. Tobiszewski, J. Namieśnik, F. Pena-Pereira, Green Chem. 2017 10.1039/c6gc03424a.

[30] S. Valencia, A. Corma, UOP LLC, United States, 1999

[31] S. Tolborg, A. Katerinopoulou, D. D. Falcone, I. Sádaba, C. M. Osmundsen, R. J. Davis, E. Taarning, P. Fristrup, M. S. Holm, J. Mater. Chem. A 2014, 2, 20252-20262. 


\section{Entry for the Table of Contents}

\section{COMMUNICATION}

A quantitative NMR approach is used to detect, quantify and optimize the formation sugar-derived building blocks without depending on reference compounds. Record yields of target molecules are achieved by obstructing competing reactions through solvent choice.

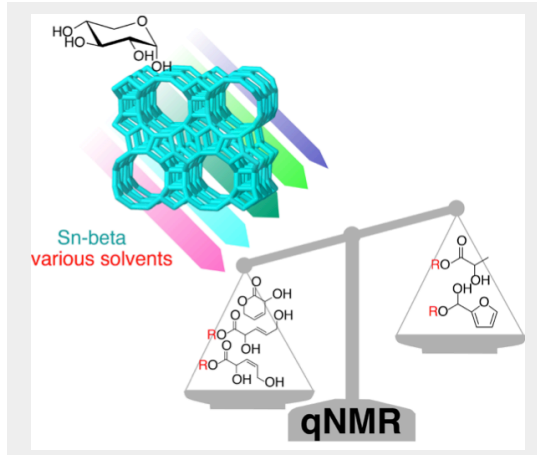

Samuel G. Elliot, Søren Tolborg, Irantzu Sádaba, Esben Taarning, and Sebastian Meier*

Page No. - Page No.

Quantitative NMR Approach to Optimize the Formation of Chemical Building Blocks from Abundant Carbohydrates 